\title{
Screening young people for obsessive-compulsive
} disorder

\author{
RUDOLF UHER, ISOBEL HEYMAN, CATHERINE MORTIMORE, \\ IAN FR AMPTON and ROBERT GOODMAN
}

\begin{abstract}
Summary Obsessive-compulsive disorder (OCD) in young people is underrecognised and undertreated. Simple screening tools suitable for general practice and community services are needed. We created a seven-item selfreport Short OCD Screener (SOCS) and administered it to young people aged II- 15 years, including 116 patients with $O C D, 18 \mid$ healthy community controls and 33 young people with other psychiatric diagnoses. The SOCS has excellent sensitivity of 0.97 (95\% Cl 0.9I-0.98) to detect OCD cases. Its specificity is good in children without psychiatric diagnoses, but low in a psychiatric sample. The SOCS is a screening tool suitable for community but not specialist settings.
\end{abstract}

\section{Declaration of interest None.}

Obsessive-compulsive disorder (OCD) commonly arises in childhood and adolescence (Heyman et al, 2001). Young people with the disorder perceive their symptoms as embarrassing and do not disclose them unless specifically asked. Therefore, OCD in this age group often remains unrecognised and untreated. The associated distress and developmental handicap are avoidable as effective treatments are available, namely cognitive-behavioural therapy with or without serotonin reuptake inhibiting medication (Heyman et al, 2006). There is evidence that early detection and intervention improve outcome (Stewart et al, 2004). The National Institute for Health and Clinical Excellence (NICE) guidance on the assessment and treatment of OCD recommends routine screening of young people at risk in general practice or other settings where they may present for help (National Collaborating Centre for Mental Health, 2005). Such screening requires short, easy-to-use and widely available measures. We report on the development, validation and dissemination of such a selfreport tool, the Short OCD Screener (SOCS).

\section{METHOD}

The questionnaire was developed from the five most discriminant items of the 44-item child version of the Leyton Obsessional Inventory (Berg et al, 1986). These items enquire about common symptoms including checking, touching, cleanliness/washing, repeating and exactness. Two further questions were designed to gauge the associated impairment and resistance. A three-option response format ('no', 'a bit', or 'a lot') was used throughout. A SOCS score is calculated by summing the scores for all seven items ('no', 0 , 'a bit', 1; 'a lot', 2).

We administered the SOCS to 127 individuals aged 11-15 years consecutively referred to the Clinic for ObsessiveCompulsive and Related Disorders at the Michael Rutter Centre, Maudsley Hospital, London. Of the 127 referred individuals, 114 met ICD-10 diagnostic criteria for OCD (World Health Organization, 1992), established by a comprehensive psychiatric assessment and the structured Child YaleBrown Obsessive-Compulsive Scale (CYBOCS; Scahill et al, 1997). The remaining 13 individuals received other ICD-10 diagnoses, including anxiety disorder $(n=7)$, conduct disorder $(n=4)$, hyperactivity $(n=3)$ and depression $(n=3)$. All participants completed the SOCS prior to clinical assessment.

We further administered the SOCS to a community sample of 203 children aged 11-15 years as a part of the British nationwide pilot survey of child and adolescent mental health (Goodman, 1999). Diagnoses of ICD-10 psychiatric disorders in the community sample were established using the Development and Well-Being Assessment (Goodman et al, 2000). Two of the individuals in this sample met diagnostic criteria for OCD and 20 had other ICD-10 diagnoses including conduct disorder $(n=12)$, anxiety disorders $(n=6)$, hyperkinetic disorder $(n=3)$ and depression $(n=2)$. The clinic and community samples were combined to obtain a group of 116 cases of OCD, including 72 boys and 44 girls with mean age 13.3 years (s.d. $=1.3$, range $11-15$ ), mean duration of illness 3.3 years (s.d.=2.2, range 0.5-10) and mean total CY-BOCS impairment score 23.1 (s.d.= 5.0, range $15-40$ ).

Three overlapping control groups were used. The first comparison group comprised the 181 individuals without any psychiatric diagnosis from the community sample, constituting the 'pure healthy control' group (mean age 13.0 years, s.d.= 1.4; 98 boys). This group was used to obtain estimates of how well the SOCS can discriminate OCD cases from healthy individuals. The second control group was also drawn from the community sample and consisted of healthy individuals and those with non-OCD psychiatric diagnoses, forming a 'mixed community control' group of 201 with a proportion of individuals with other psychiatric diagnoses representative of the general population (mean age 13.0 years, s.d. $=1.4 ; 111$ boys). This group was used to provide more realistic estimates of discrimination in a community setting. The third control group is a 'psychiatric control' group, included 33 individuals with a psychiatric diagnosis other than OCD from both the community and the clinic samples (mean age 13.1 years, s.d. $=1.3 ; 20$ boys); this group was used to explore whether the SOCS could discriminate OCD from other psychiatric disorders in clinical samples.

We used receiver operating characteristics analysis to establish optimal cut-offs for screening (Fombonne, 1991). The 95\% confidence intervals for proportions were calculated using the efficient score method (Newcombe, 1998).

\section{RESULTS}

The mean total SOCS scores were 9.7 (s.d.=2.2) for the OCD group, 3.0 (s.d.=2.3) for the healthy control group, 3.3 (s.d.=2.5) for the mixed community control group and 5.8 (s.d.=2.8) for the psychiatric control group. Thus an average OCD case scored 3 standard deviations above the healthy population mean and 1.5 standard deviations above the mean of psychiatric controls. 
Internal consistency of the SOCS was good, with Cronbach's $\alpha=0.85$. Item-total correlations were all above 0.4 . A principal axis factor analysis clearly indicated unidimensionality with a single latent factor explaining $53 \%$ of variance and all item loadings $>0.4$.

A SOCS score of 6 or more differentiated OCD cases with a sensitivity of 0.97 (95\% CI $0.91-0.99)$. The specificity was 0.88 (95\% CI 0.82-0.92) for differentiation from the healthy control group, 0.84 (95\% CI $0.78-0.89)$ for the mixed community control group, and $0.52(95 \% \mathrm{CI}$ 0.34-0.69) for the psychiatric control group. Thus the screener identifies almost all true cases of OCD, approximately one in ten healthy adolescents and one in two of those with other psychiatric disorders (Table 1). In the composite sample of OCD cases and mixed community controls, the positive predictive value was 0.78 (95\% CI $0.70-0.84$ ) and the negative predictive value was 0.98 (95\% CI 0.94-0.99).

\section{DISCUSSION}

The SOCS is shorter than other self-report tools (Bamber et al, 2002; Hudziak et al, 2006) and has comparable or better discriminant characteristics. The high sensitivity and negative predictive value make the SOCS an adequate screening tool for settings such as general practice, educational psychology, paediatric services or dermatology clinics. Because of its moderate specificity the SOCS cannot be recommended for making diagnoses in psychiatric samples.

The use of a composite sample from the general population and from referred patients allowed collection of a large sample of young people with OCD. However, this enriched sample differed from the population encountered in community practice. Obsessive-compulsive disorder was more
RUDOLF UHER, South London and Maudsley NHS Trust, Institute of Psychiatry, King's College London; ISOBEL HEYMAN, National Clinic for Young People with OCD, South London and Maudsley NHS Trust and Institute of Psychiatry, King's College London; CATHERINE MORTIMORE, Camden Primary Care Trust, London; IAN FRAMPTON, ROBERT GOODMAN, Institute of Psychiatry, King's College London, UK

Correspondence: Dr Isobel Heyman, Department of Child Psychiatry, Institute of Psychiatry, De Crespigny Park, London SE5 8AF, UK. Tel: +44 (0)20 7740 5222; fax: +44 (0)20 7740 50II ; email: i.heyman@iop.kcl.ac.uk

(First received 22 December 2006, final revision 12 March 2007, accepted 29 March 2007) prevalent in the study sample and more severe forms of the disorder might be overrepresented. A study of young people attending primary care will be needed to establish more accurate estimates of discriminatory characteristics for this setting.

The questionnaire is available online at http://ocdyouth.iop.kcl.ac.uk/downloads/ socs.pdf. We hope that this simple tool will help to implement the NICE guideline recommendation to increase the awareness and detection of OCD in young people (National Collaborating Centre for Mental Health, 2005). It is potentially suitable for use in primary care, community child health services, educational psychology or specialist medical settings where OCD is common, such as dermatology clinics (Fineberg et al, 2003). A negative result (SOCS score 5 or lower) means that OCD is unlikely. A positive result does not mean that the young person has OCD but should serve as a basis for discussion with the young person and parents, and diagnostic assessment. In our experience, young people find that completing this measure helps them with the initial stages of treatment, as it provides them with a vocabulary to use with their therapist.

\section{REFERENCES}

\section{Bamber, D., Tamplin, A., Park, R. J., et al (2002)}

Development of a short Leyton obsessional inventory for children and adolescents. Journal of the American
Table I Numbers of true and false positives/negatives using the cut-off score of 6 or more on the Short OCD Screener

\begin{tabular}{lccc}
\hline & Screen positive & Screen negative & Total \\
\hline Obsessive-compulsive disorder cases & 112 & 4 & 116 \\
Pure healthy controls & 22 & 159 & 181 \\
Mixed community controls & 32 & 169 & 201 \\
Psychiatric controls & 16 & 17 & 33 \\
\hline
\end{tabular}

Academy of Child and Adolescent Psychiatry, 4I, 12461252.

Berg, C. J., Rapoport, J. L. \& Flament, M. (1986) The Leyton Obsessional Inventory - Child Version. Journal of the American Academy of Child and Adolescent Psychiatry, 25, 84-91.

Fineberg, N. A., O'Doherty, C., Rajagopal, S., et al (2003) How common is obsessive-compulsive disorder in a dermatology outpatient clinic? Journal of Clinical Psychiatry, 64, 152-155.

Fombonne, E. (1991) The use of questionnaires in child psychiatry research: measuring their performance and choosing an optimal cut-off. Journal of Child Psychology and Psychiatry, 32, 677-693.

Goodman, R. (1999) The extended version of the Strengths and Difficulties Questionnaire as a guide to child psychiatric caseness and consequent burden. Journal of Child Psychology and Psychiatry, 40, 791-799.

Goodman, R., Ford, T., Richards, H., et al (2000) The Development and Well-Being Assessment: description and initial validation of an integrated assessment of child and adolescent psychopathology. Journal of Child Psychology and Psychiatry, 4I, 645-655.

Heyman, I., Fombonne, E., Simmons, H., et al (200I) Prevalence of obsessive-compulsive disorder in the British nationwide survey of child mental health. British Journal of Psychiatry, 179, 324-329.

Heyman, I., Mataix-Cols, D. \& Fineberg, N. A. (2006) Obsessive-compulsive disorder. BMJ, 333, 424-429.

Hudziak, J. J., Althoff, R. R., Stanger, C., et al (2006) The Obsessive Compulsive Scale of the Child Behavior Checklist predicts obsessive-compulsive disorder: a receiver operating characteristic curve analysis. Journal of Child Psychology and Psychiatry, 47, 160-166.

Newcombe, R. G. (1998) Two-sided confidence intervals for the single proportion: comparison of seven methods. Statistics in Medicine, 17, 857-872.

National Collaborating Centre for Mental Health (2005) Obsessive-Compulsive Disorder: Core Interventions in the Treatment of Obsessive Compulsive Disorder and Body Dysmorphic Disorder. British Psychological Society Royal College of Psychiatrists.

Scahill, L., Riddle, M. A., McSwiggin-Hardin, M., et al (1997) Children's Yale-Brown Obsessive Compulsive Scale: reliability and validity. Journal of the American Academy of Child and Adolescent Psychiatry, 36, 844-852

Stewart, S. E., Geller, D. A., Jenike, M., et al (2004) Long-term outcome of pediatric obsessive-compulsive disorder: a meta-analysis and qualitative review of the literature. Acta Psychiatrica Scandinavica, II0, 4-13.

World Health Organization (1992) Tenth Revision of the International Statistical Classification of Diseases and Related Health Problems (ICD-10).WHO. 